\title{
Chronic low back pain during COVID-19 lockdown: is there a paradox effect?
}

\author{
Aymeric Amelot ${ }^{1}$ (1) $\cdot$ Anais Jacquot ${ }^{2} \cdot$ Louis-Marie Terrier $^{1} \cdot$ Mourad Aggad $^{1} \cdot$ Alexia Planty-Bonjour $^{1}$. \\ Bernard Fouquet $^{2} \cdot$ Ann-Rose Cook $^{1}$ - Ilyess Zemmoura ${ }^{1}$. Stephane Velut ${ }^{1}$. Christophe Destrieux ${ }^{1}$. \\ Patrick François ${ }^{1} \cdot$ Pierre-Yves Borius ${ }^{3} \cdot$ Bertrand Mathon $^{3}$
}

Received: 22 March 2021 / Accepted: 27 October 2021 / Published online: 2 November 2021

(c) The Author(s), under exclusive licence to Springer-Verlag GmbH Germany, part of Springer Nature 2021

\begin{abstract}
Purpose The coronavirus 2019 (COVID-19) pandemic led to a compulsory lockdown of 3 months with strict restrictions. The impact of the COVID-19 pandemic has shown broad repercussions on patients with chronic pain; especially for conditions that present a significant emotional participation such as chronic low back pain (cLBP).

Methods We performed a prospective study on 50 patients. Pre- and 1-month post-lockdown questionnaires such as: the Impact of Event Scale (IES), the Oswestry Disability Index (ODI), the Roland-Morris questionnaire (RMQ) and the visual analogue scale (VAS) for back and leg pain intensity were collected.

Results The mean time of the evolution of cLBP was 33.04 months (range 5-120 months). Eighteen (36\%) patients improved their cLBP (i-cLBP), whereas for $14(28 \%)$ it was worse (w-cLBP). Cox multivariate proportional hazard model identified that MODIC 1 disc disease [OR 19.93, IC95\% (2.81-102.13), $p=0.015]$ and at-home workouts [OR 18.854, IC95\% (1.151-204.9), $p=0.040]$ were good prognosis factors of the improvement of cLBP while subclinical/mild Covid-19 anxiety (IES score <26) was a poor prognosis factor in improving cLBP [OR 0.21, IC95\% (0.001-0.384), $p=0.009$ ]. Furthermore, pre-lockdown benzodiazepine medication [OR 2.554, IC95\% (1.20-9.9), $p=0.002$ ] was a prognosis factor of worse cLBP. In contrast, patients with severe Covid-19 anxiety (IES score>26) significantly improved their cLBP [OR 0.58, IC95\% $(0.025-0.834), p=0.01]$.
\end{abstract}

Conclusion Lockdown affected the somatic component of cLBP by decreasing activities and physical measures, whereas the SARS-CoV-2 pandemic spectrum paradoxically improved the psychic and emotional component of cLBP.

Keywords COVID-19 · Containment · Pain · Chronic low back pain · Anxiety

\section{Introduction}

In March 2020, due to the rapidity of the dissemination of the new strain of severe acute respiratory syndrome coronavirus 2 (SARS-CoV-2) and the severity of the disease that it causes, the World Health Organization qualified the COVID-19 disease as a pandemic. The French government,

Aymeric Amelot

aymmed@hotmail.fr

1 Department of Neurosurgery, CHU Tours, 2 Boulevard de Tonnelle, 37000 Tours, France

2 Physical Medicine and Rehabilitation Department, CHU de Tours, Tours, France

3 Department of Neurosurgery, La Pitié-Salpêtrière, Paris, France like many other nations, declared a state of health emergency by imposing the entire country in firm lockdown. Indeed, a typical community-wide lockdown was designed to force the population to stay at home and restrict their movements in order to minimize exposure and the dissemination of this highly transmissible disease.

The impact of the ongoing pandemic, caused by SARSCoV-2, had far-reaching implications on how we deliver routine care to patients. Therefore, the management of patients with a new or previously known pain complaint can be challenging [1].

Significant psychosocial outcomes may be observed in result of the physical distancing, isolation and the changes in social behaviour and work activities, as well as the unavailability of the public health system to deliver medical management of chronic pain, in addition to the general fear of the 
infection [2, 3]. Indeed, all these factors could dramatically increase the burden of chronic pain conditions such as low back pain.

Chronic low back pain (cLBP) affects $15-30 \%$ of the population [4] and is the leading cause of disability worldwide [5]. Patients affected by cLBP require long-term multidisciplinary management even during a pandemic. Psychological variables such as fear of abandonment, anxiety and depression may increase during this period of social isolation and aggravate pain conditions. Furthermore, in certain events (i.e. natural disasters, wars, pandemics), pain dimensions have seen to be greatly impacted by the psychological status of patients [6].

We conducted a prospective study to investigate the impact of COVID-19 lockdown and the pandemic environment on chronic low back pain.

\section{Materials and methods}

\section{Ethics statement}

Data were collected according to the STROBE statement and were stored in a computer file in accordance with the law of the French Data Protection Act of 6 January 1978 amended in 2004. The protocol can be found in the reference methodology MR003 chapter adopted by the CNIL to which the University Hospital of this project conforms.

\section{Study population}

Between Mars and June 2020, the Neurosurgery and Physical Medicine and Rehabilitation departments conducted a prospective series of 50 consecutive patients treated and followed for chronic low back pain (cLBP). For all these patients, cLBP was caused by degenerative disc disease with paraspinal muscle impact. None of these patients presented other spinal lesions (spondylolisthesis, disc herniation, stenosis).

\section{Clinical outcomes}

All data were collected prior and in the first month following the end of lockdown (January-June 2020). For each patient data were recorded during a face-to-face follow-up consultation. Concerning: age, sex, work, medical history, time from onset of CLBP, body mass index (BMI), spine history, MRI MODIC disc grade, drug consumption and lockdown details (physiotherapist, home activities, analgesia, diet). The Impact of Event Scale (IES), the Oswestry Disability Index (ODI), the Roland-Morris questionnaire (RMQ) and the visual analogue scale (VAS) for back and leg pain intensity were also collected. For these different pain scales, the recovery rate taking into consideration and quantifying patient recovery in comparison with perfect recovery scores was determined by the following equation [7]: ((postoperative score-preoperative score)/(total score-preoperative score) $) \times 100$.

IES scale: the Impact of Event Scale (IES) was developed to measure current subjective distress related to a specific life event [8]. In our study, the global COVID-19 pandemic represented the specific life event. The IES has 15 items, seven of which measure intrusive symptoms such as thoughts, nightmares, feelings and images associated with the specific event. Five of these items reflect intrusive symptoms while awake and two reflect intrusion during sleep (nightmares, insomnia).

The Oswestry Disability Index (ODI): is an index derived from the Oswestry Low Back Pain questionnaire [9]. The self-completed questionnaire contains ten topics concerning intensity of pain, lifting, ability to care for oneself, ability to walk, ability to sit, sexual function, ability to stand, social life, sleep quality and the ability to travel.

The Roland-Morris questionnaire $(R M Q)$ : is a 24 -item patient-reported outcome measure that inquires pain-related disability resulting from LBP. Items are scored 0 if left blank and 1 if endorsed, for a total RMQ score ranging from 0 to 24; higher scores represent higher levels of pain-related disability [10].

\section{Statistical analysis}

All tests were two-sided; $p$-values $<0.05$ were considered statistically significant. Univariate and multivariate Cox proportional hazard regression models were conducted using SPSS software, version 22.0 (SPSS, Chicago, IL, USA). Establishment and verification of nomograms were implemented using the open-source software R-version 3.2.5 with rms packages (Design, Vienna, Austria). Pearson's Chisquare test or Fisher's exact test was applied for categorical variables. Student's t test or Wilcoxon test was used for twogroup comparisons of normally or non-normally distributed continuous variables, respectively. Logistic regression analysis was used to estimate crude odds ratios (cORs), adjusted odds ratios (aORs) and their $95 \%$ confidence intervals (CI) for possible influencing factors. A two-sided $p$ value of 0.05 or less was considered to be statistically significant.

\section{Results}

\section{Medical and demographic characteristics (Table 1)}

In our prospective series, 50 patients with chronic low back pain (cLBP) were included: $26(52 \%)$ women and $24(48 \%)$ men, with a mean age of 52.6 years (range 
Table 1 Medical and demographic characteristics of patients during lockdown. Nonsteroidal anti-inflammatory drug (NSAID) and spinal manipulative therapy (SMT)

\begin{tabular}{|c|c|}
\hline & 50 patients $(\%)$ \\
\hline \multicolumn{2}{|l|}{ Gender } \\
\hline Male & $24(52)$ \\
\hline Female & $26(48)$ \\
\hline Mean age in years (range) & $52.6(21-76)$ \\
\hline Mean time cLBP in months (range) & $33.04(5-120)$ \\
\hline BMI (range) & $26.2(18.6-40.1)$ \\
\hline \multicolumn{2}{|l|}{ Medical history } \\
\hline Cardiovascular & $19(38)$ \\
\hline Chronic disease & $9(18)$ \\
\hline Depression & $5(10)$ \\
\hline Herniated disc & $7(14)$ \\
\hline \multicolumn{2}{|l|}{ Marital status } \\
\hline In relationship & $41(82)$ \\
\hline Separated/alone & $9(18)$ \\
\hline Radiculalgia associated & $29(58)$ \\
\hline \multicolumn{2}{|l|}{ Modic disc classification } \\
\hline Type 0 & $22(40)$ \\
\hline Type I & $12(24)$ \\
\hline Type II & $16(34)$ \\
\hline \multicolumn{2}{|l|}{ Drug consumption } \\
\hline Acetaminophen & $17(34)$ \\
\hline NSAID & $31(62)$ \\
\hline Opioids & $15(30)$ \\
\hline Benzodiazepine & $15(30)$ \\
\hline Other (brace, medullar stimulation) & $3(6)$ \\
\hline \multicolumn{2}{|l|}{ Pre-containment professional activity } \\
\hline Active & $18(36)$ \\
\hline Unemployed & $10(20)$ \\
\hline Cessation of work (cLBP) & $17(34)$ \\
\hline Pensioner & $5(10)$ \\
\hline \multicolumn{2}{|l|}{ Pre-containment physical activity } \\
\hline Regular & $13(26)$ \\
\hline Physiotherapy and SMT & $27(54)$ \\
\hline \multicolumn{2}{|l|}{ Containment characters } \\
\hline Home teleworked & $5(10)$ \\
\hline No work & $44(88)$ \\
\hline House containment & $36(72)$ \\
\hline Apartment containment & $14(28)$ \\
\hline Weight gain & $21(42)$ \\
\hline Moderate physical activity (walk, bike) & $28(56)$ \\
\hline Home stretching exercises & $19(38)$ \\
\hline Increasing tobacco/alcohol consumption & $5(10)$ \\
\hline
\end{tabular}

21-76 years). Demographic data are summarized in Table 1 . The mean body mass index (BMI) was $26,2 \mathrm{~kg} / \mathrm{m}^{2}$ (range $18.6-40.1 \mathrm{~kg} / \mathrm{m}^{2}$ ). Nineteen patients (38\%) had cardiovascular medical history, $9(18 \%)$ had a chronic disease (diabetes, thyroid, pulmonary or arthritis) and 5 (10\%) a history of depression. Forty-one patients (82\%) were in a relationship and $9(18 \%)$ were single or separated. Employment status distribution concerning the active population was: $28(56 \%)$ workers, $10(20 \%)$ unemployed/ invalidated and $8(16 \%)$ managers.

\section{Pre-containment CLBP (Table 1)}

Eighteen (36\%) patients worked, $10(20 \%)$ were unemployed/invalidated, 17 (34\%) were in cessation of work due to cLBP and $5(10 \%)$ were pensioners. The mean time of the evolution of cLBP was 33.04 months (range 5-120 months). Radiculalgia was associated with cLBP in 29 patients (58\%). Seven patients (14\%) had a history of a herniated disc. Modic changes classification was as follows: 22 patients (40\%) had type 0 (normal disc and vertebral body appearance), 12 (24\%) had type I (presence of bone marrow oedema within vertebral body and hyper-vascularization) and $16(32 \%)$ had type II (fatty replacements of the red bone marrow within the vertebral body) [11]. Drug consumption was distributed as follows: 17 patients (34\%) took paracetamol/acetaminophen, 31 (62\%) nonsteroidal anti-inflammatory drugs (NSAIDs), 15 (30\%) opioid drugs such as morphine and oxycodone and 15 (30\%) took anti-epileptic/benzodiazepine drugs. In addition, three patients (6\%) used braces (2) or medullary stimulation (1).

\section{COVID-19 lockdown conditions (Table 1)}

Forty-four patients (88\%) were in full lockdown and 6 partially, 5 (10\%) teleworked. Fourteen patients (28\%) went through lockdown in an apartment and 36 (72\%) in a house with a garden. Prior lockdown, 13 (26\%) patients practiced a regular physical activity and during lockdown, and 28 patients (56\%) performed moderate physical activity such as walking outdoors.

For patients, who benefited from pre-containment physiotherapy 26/27 (96\%), had to stop and 19/27 (70.3\%) continued to perform the stretches and exercises they were taught, by themselves. The stretching exercises were mainly hyperextensions of the spinal erector muscles (inspired by the McKenzie method), rotations and flexions of the trunk, strengthening of the abdominal strap and rebalancing of the pelvis (psoas muscles, adductors, quadriceps, ischio-legs, pelvic-trochanteric and glutes).

Twenty-one patients (42\%) gained in average $1.9 \mathrm{~kg}$ (SD $2.5)$ during lockdown, and 3 patients (6\%) watched their diet. Only 2 patients (4\%) and $3(6 \%)$ confided to have increased, respectively, their tobacco and alcohol consumption. 


\section{Post-containment cLBP}

During lockdown, 25 patients $(50 \%)$ saw an increase in their consumption of analgesics, while $15(30 \%)$ patients decreased it. Eighteen (36\%) patients improved their cLBP (i-cLBP), 18 (36\%) patients saw no change, whereas for $14(28 \%)$ it became worse (w-cLBP). The mean time of cLBP evolution was 29.9 months (SD 25.6) for i-cLBP vs 32.8 months (SD 18.1) for w-cBLP, $p=0.618$.

\section{i-cLBP group (Table 2)}

The mean pre-containment ODI score for $18 \mathrm{i}$-cLBP patients was $45.8 \%$ (SD, 16.4) and decreased significantly to $19.3 \%$ $(\mathrm{SD}, 16.7)$ in the post-containment period $(p=0.001)$. Furthermore, the mean pre-containment and post-containment VAS back scores were 7.5 (SD 2.0) and 3.6 (SD 2.9), respectively $(p=0.016)$. Likewise, the pre-containment VAS leg score (5.7, SD 3.2) barely reached a post-containment score of 2.8 (SD 2.3) $(p=0.02)$. The mean pre-containment RMQ score was $44.8 \%$ (SD 18.6) and decreased significantly to $19.68 \%$ (SD, 14.8) ( $p=0.0002)$ during the post-containment period or at the post-containment visit.

\section{w-cLBP group (Table2)}

The mean pre-containment ODI score for $14 \mathrm{w}$-cLBP patients was 39.7\% (SD 20.9) and degraded significantly to $58.8 \%$ (SD 12.7) at post-containment $(p=0.001)$. The mean pre-containment and post-containment VAS back scores were 6.1 (SD 2.4) and 8.3 (SD 1.5), respectively $(p=0.03)$.

Table 2 Evolution of the pre- and post-containment functional/pain scores according to the 3 groups: $i$-cLBP, $w$-cLBP and unchanged cLBP

\begin{tabular}{llll}
\hline & Pre-containment (SD) & Post-containment (SD) & $p$ value \\
\hline$i$-cLBP $(18,36 \%)$ & & \\
ODI (\%) & $45.8(16.4)$ & $19.3(16.7)$ & $\mathbf{0 . 0 0 1}$ \\
VAS back & $7.5(2.0)$ & $3.6(2.9)$ & $\mathbf{0 . 0 1 6}$ \\
VAS leg & $5.7(3.2)$ & $2.8(2.3)$ & $\mathbf{0 . 0 2}$ \\
RMQ (\%) & $44.8(18.6)$ & $19.7(14.8)$ & $\mathbf{0 . 0 0 0 2}$ \\
-cLBP $(14,28 \%)$ & & \\
ODI (\%) & $39.7(20.9)$ & $58.8(12.7)$ & $\mathbf{0 . 0 0 1}$ \\
VAS back & $6.1(2.4)$ & $8.3(1.5)$ & $\mathbf{0 . 0 0 3}$ \\
VAS leg & $5.4(2.6)$ & $5.9(3.2)$ & 0.191 \\
RMQ (\%) & $42.1(17.9)$ & $53.2(13.9)$ & $\mathbf{0 . 0 0 0 1}$ \\
Unchanged-LBP $(18,36 \%)$ & & \\
ODI (\%) & $38.9(17.2)$ & $39.2(17.1)$ & 0.987 \\
VAS back & $7.4(1.7)$ & $6.6(2.4)$ & 0.675 \\
VAS leg & $4.9(3.2)$ & $4.5(3.4)$ & 0.345 \\
RMQ (\%) & $44.8(19.5)$ & $47.3(20.4)$ & 0.648 \\
\hline
\end{tabular}

Significant $p$ values are in bold letter
Finally, the pre-containment VAS leg score (5.4, SD 2.6) declined in post-containment: VAS leg score of 5.9 (SD 3.2) $(p=0.191)$. The mean pre-containment RMQ score was $42.1 \%$ (SD 17.9) and increased significantly to $53.21 \%$ (SD 13.9) during the post-containment period $(p=0.0001)$.

\section{Unchanged cLBP group (Table 2)}

Eighteen (36\%) patients had unchanged cLBP. Their mean pre-containment ODI score was 38.9\% (SD 17.2) and 39.2\% (SD 17.1) at post-containment $(p=0.987)$. The mean precontainment and post-containment VAS back scores were 7.4 (SD 1.7) and 6.6 (SD 2.4), respectively ( $p=0.675)$. Precontainment VAS leg score (4.9, SD 3.2) was unmodified in post-containment 4.5 (SD 3.4) $(p=0.345)$. Indeed, the mean pre-containment RMQ score was $44.8 \%$ (SD 19.5) and did not change at post-containment 47.3\% (SD 20.4) $(p=0.648)$. The IES score was 13.3 (SD 18.8).

\section{i-cLBP vs w-cLBP group evolution}

There were no statistical differences between the two groups (successively $\mathrm{i}$-cLBP and $\mathrm{w}$-cLBP) for pre-containment scores and data: ODI $p=0.349$; mean RMQ score $p=0.761$; mean VAS back $p=0.499$; mean VAS leg $p=0.772$, mean pre-containment BMI: 27.2 (SD 6.3) versus 24.7 (SD 3.9), $p=0.211$ and gender ratio $p=0.247$.

The mean RMQ improvement rate after containment was $61.3 \%$ (SD 48.4) for $\mathrm{i}$-cLBP versus $-15.1 \%$ (SD 21.6) $(p<0.0001)$. Similarly, improvements were appreciated thanks to the ODI with a recovery rate of $60.6 \%$ (SD 46.8 ) for $\mathrm{i}$-cLBP versus $-29.0 \%$ (SD 16.1) for w-cLBP $(p<0.0001)$. Respectively, the EVA-back improvement rate was $88.2 \%$ (SD 41.9) for i-cLBP versus $-54.9 \%$ (SD 27.2) for w-cLBP $(p<0.0001)$ and the EVA-leg improvement rate was $61.2 \%$ (SD 34.2) versus $-40.2 \%$ (SD 42.4) for w-cLBP $(p=0.0001)$. The IES score centred on the COVID-19 pandemic was close to 50.4 (SD 18.1) for i-cLBP and 13.4 (SD 11.2) for $\mathrm{w}$-cLBP $(p=0.001)$.

\section{CLBP prognosis factors during containment (Tables 3 and 4)}

We sought to identify prognostic factors associated with the improvement and the worsening of cLBP using a univariate analysis. The results are presented in Table 3. Indeed, patients with modic type 1 disc disease $(p<0.0001)$, house containment $(p=0.03)$, home self-physiotherapist exercises $(p=0.003)$, lighter diet $(p=0.042)$ and high Covid-19 anxiety (IES $>26)(p<0.0001)$ were statistically associated with an improvement in cLBP.

Cox multivariate proportional hazard model identified that MODIC 1 disc disease [OR 19.93, IC95\% 
Table 3 Univariate ( $p$ value was calculated by the log-rank test) and multivariate ( $p$ value was calculated by Cox regression) analysis for prognostic factors of cLBP improvement (i-cLBP)

\begin{tabular}{|c|c|c|c|}
\hline & $\begin{array}{l}\text { Univariate analysis } \\
(p)\end{array}$ & $\begin{array}{l}\text { Multivariate analysis } \\
(p)\end{array}$ & OR (CI 95\%) \\
\hline \multicolumn{4}{|l|}{ Gender } \\
\hline Male/female & 0.724 & & \\
\hline \multicolumn{4}{|l|}{ Age } \\
\hline$<50$ y.o & 0.556 & & \\
\hline$<65$ y.o & 0.724 & & \\
\hline cLBP time evolution & 0.388 & & \\
\hline $\mathrm{BMI}>25$ & 0.774 & & \\
\hline $\mathrm{BMI}>30$ & 0.156 & & \\
\hline \multicolumn{4}{|l|}{ Medical history } \\
\hline Cardiovascular & 0.552 & & \\
\hline Chronic disease & 0.618 & & \\
\hline Depression & 0.326 & & \\
\hline Herniated disc & 0.987 & & \\
\hline Marital status & 0.130 & & \\
\hline Radiculalgia associated & 0.149 & & \\
\hline Subclinical/mild IES & $<0.0001$ & 0.009 & $0.21(0.001-0.384)$ \\
\hline \multicolumn{4}{|l|}{ Modic disc classification } \\
\hline Type 0 & 0.250 & & \\
\hline Type I & $<0.0001$ & 0.015 & $19.93(2.81-102.13)$ \\
\hline Type II & 0.621 & & \\
\hline \multicolumn{4}{|l|}{ Drug consumption } \\
\hline Acetaminophen & 0.336 & & \\
\hline NSAID & 0.552 & & \\
\hline Opioids & 0.370 & & \\
\hline Benzodiazepine & 0.348 & & \\
\hline Other & 0.398 & & \\
\hline \multicolumn{4}{|l|}{ Pre-containment work } \\
\hline Active & 0.139 & & \\
\hline Cessation of work & 1.0 & & \\
\hline \multicolumn{4}{|c|}{ Pre-containment physical activity } \\
\hline Regular & 1.00 & & \\
\hline Physiotherapy and SMT & 1.00 & & \\
\hline \multicolumn{4}{|l|}{ Containment characters } \\
\hline Home teleworked & 0.635 & & \\
\hline Stop working & 0.399 & & \\
\hline House containment & $\mathbf{0 . 0 3}$ & 0.637 & $5.87(1.32-12.26)$ \\
\hline Weight gain & 0.149 & & \\
\hline Moderate physical activity & 1.00 & & \\
\hline Home stretching exercises & $\mathbf{0 . 0 3}$ & 0.04 & $18.85(1.151-204.9)$ \\
\hline Increasing tobacco/alcohol & 0.125 & & \\
\hline
\end{tabular}

Significant $p$ values are in bold letter
(2.81-102.13), $p=0.015]$, home self-exercise practice [OR 18.854, IC95\% (1.151-204.9), $p=0.040$ ] and severe Covid-19 stress (IES score > 26 [OR 0.58, IC95\% (0.025-0.834), $p=0.01]$ were good prognosis factors of the improvement of cLBP while low Covid-19 anxiety (IES score $<26$ ) was a poor prognosis factor of the improvement in cLBP [OR 0.21, IC95\% (0.001-0.384), $p=0.009$ ]

Patients receiving pre-containment benzodiazepine medication [OR 2.554, IC95\% (1.20-9.9), $p=0.002$ ] and the absence of home self-stretching exercises [OR 4.902, IC95\% (2.47-31.3), $p=0.02$ ] were prognosis factors of worse cLBP. 
Table 4 Univariate ( $p$ value was calculated by the log-rank test) and multivariate ( $p$ value was calculated by Cox regression) analysis for prognostic factors of cLBP degradation (w-cLBP)

\begin{tabular}{|c|c|c|c|}
\hline & $\begin{array}{l}\text { Univariate analysis } \\
(p)\end{array}$ & $\begin{array}{l}\text { Multivariate analysis } \\
(p)\end{array}$ & OR (CI 95\%) \\
\hline \multicolumn{4}{|l|}{ Gender } \\
\hline Male/female & 0.533 & & \\
\hline \multicolumn{4}{|l|}{ Age } \\
\hline$<50$ у.о & 0.522 & & \\
\hline$<65$ y.o & 0.705 & & \\
\hline cLBP time evolution & 1.0 & & \\
\hline $\mathrm{BMI}>25$ & 0.534 & & \\
\hline $\mathrm{BMI}>30$ & 0.143 & & \\
\hline \multicolumn{4}{|l|}{ Medical history } \\
\hline Cardiovascular & 0.06 & & \\
\hline Chronic disease & 1.0 & & \\
\hline Depression & 0.303 & & \\
\hline Herniated disc & 0.476 & & \\
\hline Marital status & 1.0 & & \\
\hline Radiculalgia associated & 1.0 & & \\
\hline Severe IES score $>26$ & 0.01 & 0.01 & $0.21(0.025-0.834)$ \\
\hline \multicolumn{4}{|l|}{ Modic disc classification } \\
\hline Type 0 & 0.09 & & \\
\hline Type I & 0.008 & 0.357 & 0.000 (NA) \\
\hline Type II & 0.168 & & \\
\hline \multicolumn{4}{|l|}{ Drug consumption } \\
\hline Acetaminophen & 0.304 & & \\
\hline NSAID & 0.197 & & \\
\hline Opioids & 0.758 & & \\
\hline Benzodiazepine & 0.003 & 0.002 & $2.554(1.20-9.9)$ \\
\hline Other & 1.0 & & \\
\hline \multicolumn{4}{|l|}{ Pre-containment work } \\
\hline Active & 0.744 & & \\
\hline Cessation of work & 0.331 & & \\
\hline \multicolumn{4}{|c|}{ Pre-containment physical activity } \\
\hline Regular & 0.729 & & \\
\hline Physiotherapy and SMT & 0.533 & & \\
\hline \multicolumn{4}{|l|}{ Containment characters } \\
\hline Home teleworked & 0.553 & & \\
\hline Stop working & 1.00 & & \\
\hline House containment & 1.00 & & \\
\hline Weight gain & 0.06 & & \\
\hline Moderate physical activity & 1.00 & & \\
\hline No home stretching exercises & 0.026 & 0.02 & $4.902(2.47-31.3)$ \\
\hline Increasing tobacco/alcohol & 0.019 & 0.999 & $0.000(\mathrm{NA})$ \\
\hline
\end{tabular}

Significant $p$ values are in bold letter

\section{Discussion}

The COVID-19 pandemic and the consequent lockdown had unprecedented repercussions at both a macrosocial (economy and policies) and microsocial levels (psychological and relational well-being of citizens) $[12,13]$.
Therefore, it is conceivable that all these events are likely to have an impact on people's lives physically, emotionally and psychologically therefore having an impact on chronic pain. Chronic pain is characterized by a participation of a biopsychosocial dimension, which greatly impacts the evolution of the disease. According to the World Health Organization (WHO), cLBP has been the leading cause of 
years lived with disability (YLDs) since 1990 and remains a significant global public health concern [14]. Furthermore, $85-95 \%$ of people presenting to primary care providers do not have a specific identifiable pathoanatomical origin for their pain [15]. Moreover, it is also one of the main pathologies responsible for occupational disease and absenteeism at work [16].

Consequently, lockdown may have an impact on the pain of cLBP patients in many aspects, which include: i) increased anxiety and anger; ii) decreased outdoor exercise; and iii) stress and depression, which can trigger unhealthy dietary habits in patients.

In our study we identified that regular at-home workouts improved cLBP. This is concordant with the literature, since in adult cLBP, exercise routines have shown to be more effective in reducing pain compared with non-exercise treatments [17]. Similarly, pairwise meta-analyses examining specific kinds of exercises have shown that simple home workouts such as pilates, motor control stabilization and yoga may be more effective in reducing pain than nonexercise training comparators [18-20]. Moreover, Owen et al. concluded that workouts may also be more effective than hand-on therapist treatments [21]. The effectiveness of spinal manipulative therapy (SMT) performed by physiotherapists in the treatment of cLBP as well as the recommendations in international guidelines for the use of non-drug interventions in the treatment of cLBP remains debated [22]: in some countries, SMT is considered a firstline treatment option [23], whereas in other countries it is recommended as a component of a broader treatment package including exercise [24]. The most recent summary of these guidelines suggests that SMT should be considered a second-line or adjuvant treatment option, after exercise or cognitive behavioural therapy [25]. In our study, people who benefited from pre-containment SMT with a physiotherapist did not increase nor improve their cLBP at the standstill imposed by confinement.

The mechanism could be twofolded, as the Chinese study points out. Indeed, Yang et al. found in their Chinese series that regular physical at-home workouts were significantly related to a decrease in the risk of developing depression and anxiety symptoms [26]. Regular physical exercise might reduce the risk of mental disorders that occur during the pandemic [27] and therefore decrease the emotional and psychological components of pain. We believe that during containment, many people have forced themselves to perform stretching and physiotherapy exercises at home, something they did not do before.

Nevertheless, Sagat et al., who focused on the Saudi population in their study, determined that the containment led to a significant increase in LBP intensity among adults residing in Riyadh. Similarly, LBP prevalence increased from 38.8 to $43.8 \%$ [28]. However, there were many important biases in this study: i) the studied population did not suffer from chronic but rather acute pain, ii) data concerned prospective feelings or pain iii) and the self-administered structured questionnaire was not conducted in accordance with reference functional scores (ODI, EQ5DL, etc.).

On the other hand, in the Italian study, Borsa et al. collected data from 4 hospitals in Milan concerning LBP in the period from March to April 2020 and showed a clear reduction $(-87.2 \%)$ of patients presenting with LBP. They suggested that the decrease in visits was probably related to the fear of being exposed to SARS-CoV-2 [29].

We observed the same kind of mechanism for other pathologies, e.g. for headache evolution, Delussi et al. suggested that during lockdown, patients showed an improvement in the clinical features of their migraine, probably attributable to the distress caused by the pandemic. Indeed, the fear of being contaminated may favour a defensive behaviour, potentially improving migraines [30]

There is preliminary evidence suggesting that telerehab interventions are beneficial in reducing pain as well as improving physical functions in patients affected by nonmalignant musculoskeletal conditions such as neck and low back pain, lumbar stenosis and osteoarthritis [28].

Telerehab interventions have proven to be beneficial for patients suffering from cLBP by helping to reduce pain levels and by maintaining that improvement via booster sessions delivered through a mobile phone application and videoconferencing [31, 32].

As surprisingly demonstrated in our study, the more stressed and anxious patients were about the COVID-19 pandemic, the less functionally troublesome the cLBP pain became.

A mechanistic contributor related to the experience of cLBP is pain catastrophizing. Pain catastrophizing is a pattern of negative cognitive-emotional responses to pain that includes rumination, magnification and helplessness [33]. It has been shown to be associated with pain severity, disability and poor outcomes for patients with cLBP [34, 35]. During the containment, the patients verbalized it very well "COVID-19 is much more serious and awful than my low-back pain" or "I was afraid of dying", and therefore, cLBP was relegated into the background. Several questions may arise from this study: i) could the anxiety generated by Covid-19 distract patients' attention, by impairing their ability to focus on their cLBP which therefore seems less stressful in comparison with the pandemic? or ii) could the stress of covid-19 be a mechanism for relativizing their pathology? To answer this question, it would be necessary to include more patients in a prospective manner and to study the cLBP on more "light" confinements such as those of the second and third waves, with less stress (hope for vaccines) or amplified stress (risk of resistance of COVID-19 variants). 
As expected, we identified that MODIC 1 disc disease was a good prognosis factor of improvement in contrast to other MODIC scores. It has been well described that low back pain was consistent with the inflammatory stage of the disc disease and therefore the MODIC 1 stage (endplate oedema with alteration of bone trabeculae). The evolution in MODIC 2 marks a post-inflammatory stage, a sequellar condition, the natural history of disc senescence, less scalable with endplate fatty involution, which could explain the fact that MODIC 1 can regress and improve, while MODIC-2 cannot do so as much.

It remains difficult to interpret the consumption of benzodiazepine medication in this particular context. The patients who consumed these drugs probably presented an important psychoaffective dimension in their cLBP, and the confinement did not "a priori" improve their anxiety, perhaps due to external factors (stopping outings, couple problems, etc. ). On the contrary, work cessation for some and the telework set-up for others may have removed some stress factors for certain patients (hierarchy, performance, etc.)

Thus, to answer all the questions raised by our work, we continue to follow these patients and discuss adding environmental dimension assessments to remove bias and have a more holistic approach to cLBP.

\section{Conclusion}

The somatic component of cLBP was affected by lockdown. Indeed, our study provided evidence that physical exercise was effective in decreasing this condition and should be implemented, even increased in order to improve pain, physical function and muscle strength during lockdown. Furthermore, cLBP caused by reversible inflammatory disc disease (MODIC 1) often improves, while it is often too late once the inflammatory stage has passed and a sequellar stage has set in (MODIC 2).

The psychic and emotional component of cLBP was paradoxically improved by the stress and anxiety generated by SARS-CoV-2. We assume that the "life-threatening" stress of virus contamination may have "eclipsed" or altered the painful perception of cLBP. This study made it possible objectify more clearly the inconstant and important participation of the emotional and psychic component in the cLBP. As a result, for cLBP patients we can recognize why remedies such as hypnosis or behavioural therapies could have a beneficial effect in selected patients.

\section{Declarations}

Conflict of interest The authors report no conflict of interest.

\section{References}

1. Wahezi SE, Duarte RV, Yerra S et al (2020) Telemedicine during COVID-19 and beyond: a practical guide and best practices multidisciplinary approach for the orthopedic and neurologic pain physical examination. Pain Phys 23:S205-S238

2. Gautam M, Thakrar A, Akinyemi E, Mahr G (2020) Current and future challenges in the delivery of mental healthcare during COVID-19. SN Compr Clin Med. https://doi.org/10.1007/ s42399-020-00348-3

3. Szperka CL, Ailani J, Barmherzig R et al (2020) Migraine care in the era of covid-19: clinical pearls and plea to insurers. Headache 60:833-842. https://doi.org/10.1111/head.13810

4. Deyo R, Mirza S, Martin B (2006) Back pain prevalence and visit rates: estimates from U.S. national surveys, 2002. In: Spine. https://pubmed.ncbi.nlm.nih.gov/17077742/. Accessed 11 Dec 2020

5. Ehrlich G (2003) Low back pain. In: Bull. World Health Organ. https://pubmed.ncbi.nlm.nih.gov/14710509/. Accessed 11 Dec 2020

6. Guetti C, Angeletti C, Paladini A et al (2013) Pain and natural disaster. In: Pain Pract. Off. J. World Inst. Pain. https://pubmed. ncbi.nlm.nih.gov/23241164/. Accessed 11 Dec 2020

7. Vitzthum H-E, Dalitz K (2007) Analysis of five specific scores for cervical spondylogenic myelopathy. Eur Spine J Off Publ Eur Spine Soc Eur Spinal Deform Soc Eur Sect Cerv Spine Res Soc 16:2096-2103. https://doi.org/10.1007/s00586-007-0512-x

8. Horowitz M, Wilner N, Alvarez W (1979) Impact of event scale: a measure of subjective stress. Psychosom Med 41:209-218. https:// doi.org/10.1097/00006842-197905000-00004

9. Fairbank JC, Pynsent PB (2000). The oswestry disability index. Spine 25:2940-2952; discussion 2952. https://doi.org/10.1097/ 00007632-200011150-00017

10. Roland M, Morris R (1983) A study of the natural history of back pain. part I: development of a reliable and sensitive measure of disability in low-back pain. Spine 8:141-144. https://doi.org/10. 1097/00007632-198303000-00004

11. Modic MT, Steinberg PM, Ross JS et al (1988) Degenerative disk disease: assessment of changes in vertebral body marrow with MR imaging. Radiology 166:193-199. https://doi.org/10.1148/radio logy.166.1.3336678

12. Kisely S, Warren N, McMahon L et al (2020) Occurrence, prevention, and management of the psychological effects of emerging virus outbreaks on healthcare workers: rapid review and metaanalysis. BMJ 369:m1642. https://doi.org/10.1136/bmj.m1642

13. Brooks SK, Webster RK, Smith LE et al (2020) The psychological impact of quarantine and how to reduce it: rapid review of the evidence. Lancet Lond Engl 395:912-920. https://doi.org/10. 1016/S0140-6736(20)30460-8

14. Wu A, March L, Zheng X et al (2020) Global low back pain prevalence and years lived with disability from 1990 to 2017: estimates from the global burden of disease study 2017. Ann Transl Med 8:299. https://doi.org/10.2103/atm.2020.02.175

15. Finucane LM, Downie A, Mercer C et al (2020) International framework for red flags for potential serious spinal pathologies. J Orthop Sports Phys Ther 50:350-372. https://doi.org/10.2519/ jospt.2020.9971

16. Poulain C, Kernéis S, Rozenberg S et al (2010) Long-term return to work after a functional restoration program for chronic 
low-back pain patients: a prospective study. Eur Spine J Off Publ Eur Spine Soc Eur Spinal Deform Soc Eur Sect Cerv Spine Res Soc 19:1153-1161. https://doi.org/10.1007/s00586-010-1361-6

17. Searle A, Spink M, Ho A, Chuter V (2015) Exercise interventions for the treatment of chronic low back pain: a systematic review and meta-analysis of randomised controlled trials. In: Clin Rehabil. https://pubmed.ncbi.nlm.nih.gov/25681408/. Accessed 11 Dec 2020

18. Saragiotto B, Maher C, Yamato T et al (2016) Motor control exercise for nonspecific low back pain: a cochrane review. In: Spine. https://pubmed.ncbi.nlm.nih.gov/27128390/. Accessed 11 Dec 2020

19. Rackwitz B, de Bie R, Limm H et al (2006) Segmental stabilizing exercises and low back pain. What is the evidence? A systematic review of randomized controlled trials. In: Clin. Rehabil. https:// pubmed.ncbi.nlm.nih.gov/16894798/. Accessed 11 Dec 2020

20. Wieland L, Skoetz N, Pilkington K et al (2017) Yoga treatment for chronic non-specific low back pain. In: Cochrane Database Syst Rev https://pubmed.ncbi.nlm.nih.gov/28076926/. Accessed 11 Dec 2020

21. Owen P, Miller C, Mundell N et al (2020) Which specific modes of exercise training are most effective for treating low back pain? Network meta-analysis. In: Br. J. Sports Med. https://pubmed. ncbi.nlm.nih.gov/31666220/. Accessed 11 Dec 2020

22. Koes B, van Tulder M, Lin C et al (2010) An updated overview of clinical guidelines for the management of non-specific low back pain in primary care. In: Eur Spine J Off Publ Eur Spine Soc Eur Spinal Deform Soc Eur Sect Cerv Spine Res Soc https://pubmed. ncbi.nlm.nih.gov/20602122/. Accessed 11 Dec 2020

23. Qaseem A, Wilt T, McLean R, Forciea M (2017) Noninvasive treatments for acute, subacute, and chronic low back pain: a Clinical Practice Guideline From the American College of Physicians. In: Ann Intern Med. https://pubmed.ncbi.nlm.nih.gov/28192789/. Accessed 11 Dec 2020

24. London L (2020) Low back pain and sciatica in over 16s: assessment and management. In: PubMed. https://pubmed.ncbi.nlm.nih. gov/33090750/. Accessed 11 Dec 2020

25. Foster N, Anema J, Cherkin D et al (2018) Prevention and treatment of low back pain: evidence, challenges, and promising directions. In: Lancet Lond Engl https://pubmed.ncbi.nlm.nih.gov/ 29573872/. Accessed 11 Dec 2020

26. Yang S, Lin H, Zhu J et al (2020) Depression and anxiety symptoms among returning workers during the COVID-19 period in East China. Soc Psychiatry Psychiatr Epidemiol. https://doi.org/ 10.1007/s00127-020-01983-w

27. Yoshikawa E, Nishi D, Matsuoka YJ (2016) Association between regular physical exercise and depressive symptoms mediated through social support and resilience in Japanese company workers: a cross-sectional study. BMC Public Health 16:553. https:// doi.org/10.1186/s12889-016-3251-2

28. Peter S, P B, P PG, et al (2020) Impact of COVID-19quarantine on low back pain intensity, prevalence, and associated risk factors among adult citizens residing in riyadh (Saudi Arabia): a cross-sectional study. In: Int. J Environ Res Public Health. https:// pubmed.ncbi.nlm.nih.gov/33036287/. Accessed 11 Dec 2020

29. Borsa S, Pluderi M, Carrabba G et al (2020). Letter to the Editor: impact of COVID-19 outbreak on acute low back pain. In: World Neurosurg. https://pubmed.ncbi.nlm.nih.gov/32479910/. Accessed 11 Dec 2020

30. Delussi M, Gentile E, Coppola G et al (2020) Investigating the effects of COVID-19 quarantine in migraine: an observational cross-sectional study from the Italian national headache registry (RICe). Front Neurol 11:597881. https://doi.org/10.3389/fneur. 2020.597881

31. Peterson S (2018) Telerehabilitation booster sessions and remote patient monitoring in the management of chronic low back pain: A case series. In: Physiother Theory Pract https://pubmed.ncbi. nlm.nih.gov/29125371/. Accessed 11 Dec 2020

32. Ms H, N A, L L et al (2017) Telehealth Versus In-Person Acceptance and Commitment Therapy for Chronic Pain: A Randomized Noninferiority Trial. In: J Pain Off J Am Pain Soc. https://pubmed. ncbi.nlm.nih.gov/27838498/. Accessed 11 Dec 2020

33. Cano A, Leonard M, Franz A (2005) The significant other version of the Pain Catastrophizing Scale (PCS-S): preliminary validation. In: Pain https://pubmed.ncbi.nlm.nih.gov/16298062/. Accessed 11 Dec 2020

34. Mok L, Lee I (2008) Anxiety, depression and pain intensity in patients with low back pain who are admitted to acute care hospitals. In: J Clin Nurs. https://pubmed.ncbi.nlm.nih.gov/18298508/. Accessed 11 Dec 2020

35. Nicholas M, Linton S, Watson P, Main C (2011) Early identification and management of psychological risk factors ("yellow flags") in patients with low back pain: a reappraisal. In: Phys Ther. https://pubmed.ncbi.nlm.nih.gov/21451099/. Accessed 11 Dec 2020

Publisher's Note Springer Nature remains neutral with regard to jurisdictional claims in published maps and institutional affiliations. 